\title{
A prospective phase II study of multimodal prophylactic treatment for afatinib-induced adverse events in advanced non-small cell lung cancer (Niigata Lung Cancer Treatment Group 1401)
}

\author{
Masaaki Okajima ${ }^{1}$, Satoru Miura ${ }^{2}$, Satoshi Watanabe ${ }^{3}$, Hiroshi Tanaka ${ }^{2}$, Kazuhiko Ito ${ }^{4}$, Takashi Ishida ${ }^{5}$, \\ Masato Makino $^{6}$, Akira Iwashima ${ }^{7}$, Naoya Matsumoto ${ }^{8}$, Kazuhiro Sato ${ }^{9}$, Kosuke Ichikawa ${ }^{3}$, Tetsuya Abe ${ }^{4}$, \\ Hirohisa Yoshizawa ${ }^{10}$, Toshiaki Kikuchi ${ }^{3}$ \\ ${ }^{1}$ Department of Respiratory Medicine, Saiseikai Niigata Hospital, Niigata, Japan; ${ }^{2}$ Department of Internal Medicine, Niigata Cancer Center \\ Hospital, Niigata, Japan; ${ }^{3}$ Department of Respiratory Medicine and Infectious Diseases, Niigata University Graduate School of Medical and Dental \\ Sciences, Niigata, Japan; ${ }^{4}$ Department of Respiratory Medicine, Niigata City General Hospital, Niigata, Japan; ${ }^{5}$ Department of Internal Medicine, \\ Niigata Prefectural Central Hospital, Joetsu, Japan; ${ }^{6}$ Department of Internal Medicine, Niigata Prefectural Shibata Hospital, Shibata, Japan; \\ ${ }^{7}$ Department of Respiratory Medicine, Nagaoka Chuo General Hospital, Nagaoka, Japan; ${ }^{8}$ Department of Respiratory Medicine, Nishi-Niigata \\ Chuo National Hospital, Niigata, Japan; ${ }^{9}$ Department of Respiratory Medicine, Nagaoka Red Cross Hospital, Nagaoka, Japan; ${ }^{10}$ Department of \\ Respiratory Medicine, Niigata Medical Center Hospital, Niigata, Japan \\ Contributions: (I) Conception and design: M Okajima, S Miura, S Watanabe, H Tanaka, T Kikuchi; (II) Administrative support: S Watanabe, H \\ Yoshizawa, T Kikuchi; (III) Provision of study materials or patients: All authors; (IV) Collection and assembly of data: M Okajima, S Miura, S \\ Watanabe; (V) Data analysis and interpretation: M Okajima, S Miura, S Watanabe, H Tanaka, T Kikuchi; (VI) Manuscript writing: All authors; (VII) \\ Final approval of manuscript: All authors. \\ Correspondence to: Satoru Miura, MD, PhD. Department of Internal Medicine, Niigata Cancer Center Hospital, 2-15-3, Kawagishi-cho, Chuo-ku, \\ Niigata City 951-8566, Japan. Email: miusat1118@niigata-cc.jp.
}

Background Afatinib has shown clinical benefits in patients with non-small cell lung cancer (NSCLC) harboring epidermal growth factor receptor (EGFR) mutations. Many patients treated with afatinib experience skin or gastrointestinal toxicity. However, an effective management strategy has not been established. This prospective study was conducted to evaluate the efficacy of multimodal prophylactic treatment for afatinib-induced toxicity.

Methods: This single-arm prospective study was conducted to evaluate the efficacy of multimodal prophylactic treatment for afatinib-induced toxicity in patients with EGFR mutation positive advanced NSCLC who planned to receive a $40 \mathrm{mg}$ dose of afatinib. Eligible patients were treated with oral loperamide ( $2 \mathrm{mg}$ twice per day), prophylactic minocycline (100 mg once per day), topical medium-class steroids, and gargling with sodium azulene. The primary endpoint was the ability of prophylactic loperamide to prevent severe or intolerable diarrhea during the 4 weeks after the initial administration of afatinib. The incidence, severity and time to occurrence of diarrhea, rash, oral mucositis and paronychia were evaluated based on a daily patient questionnaire.

Results: Forty-six patients were enrolled. The primary endpoint analysis was performed in 35 patients as the per-protocol (PP) population. The 4-week successful prophylaxis rate for severe or intolerable diarrhea was $82.9 \%$ (90\% confidence interval: $70.1-91.9 \%)$. In the total population, the incidences of grade 3 or higher rash, oral mucositis and paronychia within 4 weeks were $4 \%, 2 \%$ and $4 \%$, respectively.

Conclusions: Prophylactic loperamide administration was not effective in preventing severe or intolerable diarrhea during afatinib treatment. Adequate dose reduction will be a better approach to manage afatinibinduced diarrhea. Multimodal prevention using minocycline, topical steroids and gargling with sodium azulene may be helpful to maintain compliance with afatinib treatment (UMIN000016167).

Keywords: Non-small cell lung cancer (NSCLC); epidermal growth factor receptor (EGFR); afatinib; adverse event management 
Submitted May 11, 2020. Accepted for publication Nov 01, 2020.

doi: $10.21037 /$ tlcr-20-649

View this article at: http://dx.doi.org/10.21037/tlcr-20-649

\section{Introduction}

Epidermal growth factor receptor $(E G F R)$ is the key target of driver-based precision medicine in non-small cell lung cancer (NSCLC) therapy. Mutation of the EGFR gene initiates the activation of an intracellular signaling cascade. This activation induces cell proliferation, angiogenesis and cancer metastases. EGFR tyrosine kinase inhibitors (EGFR-TKIs) inhibit these activation signals and induce a dramatic clinical response in EGFR-mutated NSCLC patients. Afatinib is categorized as a second-generation EGFR-TKI that irreversibly inhibits the ErbB family, including the EGFR tyrosine kinase domain. This agent has been approved and has shown superiority compared with chemotherapy as a first-line treatment in NSCLC patients with EGFR mutations (LUX-Lung 3 and LUXLung 6) $(1,2)$. In the combined analysis of these two trials, afatinib demonstrated a survival benefit compared with platinum-doublet chemotherapy (3). Additionally, the LUX-Lung 7 trial was the first head-to-head randomized study to compare first-generation and second-generation EGFR-TKIs (4). Progression-free survival (PFS) and timeto-treatment failure were obviously longer in the afatinib arm than in the gefitinib arm. These studies suggested that afatinib is the most attractive agent for chemo-naïve NSLC patients with EGFR mutations.

Concerning side effects, the drug-associated adverse events (AEs) diarrhea, rash, oral mucositis and paronychia were more frequent with afatinib therapy than with gefitinib or erlotinib (5). Although it has been demonstrated that these kinds of AEs do not affect quality of life in clinical trials, they are often a problem for patients in clinical practice (6). A prospective randomized phase III trial of prophylaxis for erlotinib-induced rash named the Pan Canadian Rash Trial has already been reported (7). This trial suggested that prophylactic minocycline and reactive topical steroid treatments were some of the options for the management of erlotinibinduced rash. However, investigations of preventive treatment for other AEs have been insufficient, and effective preventive treatments for various AEs induced by afatinib have not yet been established. We considered that a multimodal intervention will be needed for efficacious management to control afatinib-induced AEs. The aim of this trial is to investigate whether multimodal prophylactic supportive therapy can control AEs induced by afatinib. We present the following article in accordance with the TREND reporting checklist (available at http://dx.doi. org/10.21037/tlcr-20-649).

\section{Methods}

\section{Study design}

This study [Niigata Lung Cancer Treatment Group (NLCTG)1401] is a multicenter, single-arm prospective study evaluating the efficacy of a multimodal prophylactic intervention for advanced EGFR-mutated NSCLC patients treated with afatinib. All participants were recruited from 10 Japanese institutions according to the inclusion and exclusion criteria. Eligible patients were initiated at a dose of $40 \mathrm{mg}$ of afatinib per day with the protocol-defined multimodal prophylactic interventions. This study was performed in accordance with the Helsinki Declaration (as revised in 2013). The Institutional Review Board of each participating institution approved the protocol. This study was registered in January 2015 on the clinical trial site of the University Hospital Medical Information Network Clinical Trials Registry (registration number: UMIN000016167).

\section{Patient eligibility}

The eligibility criteria were as follows: histologically or cytologically confirmed advanced NSCLC with EGFR active mutations; an Eastern Cooperative Oncology Group (ECOG) performance status (PS) of 0 to 2; afatinib-naïve patients who planned to be treated with a $40 \mathrm{mg}$ dose of afatinib; over 20 years of age; and adequate organ function. The main exclusion criteria were as follows: history of interstitial pneumonia, active double cancer, and ileus or intestinal paralysis. All patients gave written informed consent prior to enrollment.

\section{Study objectives and statistical analysis}

The primary endpoint of this study was the ability of 


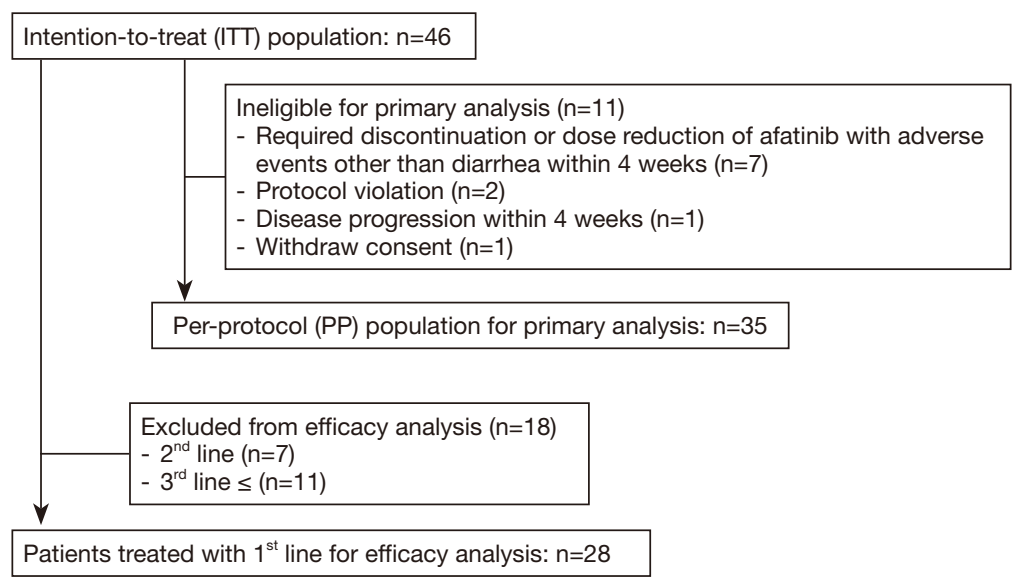

Figure 1 Details of the intent-to-treat population, per-protocol population and $1^{\text {st }}$-line efficacy analysis population.

prophylactic loperamide to prevent severe or intolerable diarrhea during the 4 weeks after the initial administration of afatinib. In the Japanese subgroup of the LUX-Lung 3 trial, the incidence of grade 3 diarrhea was $22 \%$, and the percentage of dose reductions due to diarrhea was $22.2 \%$ (8). Based on these data, the prevention rate of severe or intolerable diarrhea during 4 weeks after initiation of afatinib was calculated as $77.8 \%$. It was assumed that $75 \%$ of patients did not experience severe or intolerable diarrhea for 4 weeks. Additionally, it was assumed that if patients did receive multimodal prophylactic treatments, $90 \%$ of them would experience no severe or intolerable diarrhea for 4 weeks. With $\alpha$ and $\beta$ errors of 0.1 and 0.2 , respectively, the number of patients needed to analyze the effect of prophylactic loperamide was calculated to be 28 patients. First, we planned the target number of patients to be 30 patients, but early drop-out occurred due to side effects other than diarrhea or disease progression before 4 weeks after afatinib initiation. Thus, to obtain the analyzable cases, we set the final target number of patients as 45 . The secondary endpoints were the incidence, severity and time to onset of diarrhea, rash, mucositis and paronychia. The antitumor effects of afatinib in the first-line setting were also evaluated. We used three data sets to analyze each endpoint (Figure 1). The intent-to-treat (ITT) population was defined as all enrolled patients. The per-protocol (PP) population was defined as a group of patients excluding early drop-out cases that were not ineligible for evaluating the primary endpoint. The first-line population was defined as a group of patients who were treated with afatinib as a first-line therapy. The antitumor effect of afatinib was evaluated only in this first-line population.

\section{Patient assessment}

Evaluation of AEs induced by afatinib was based on daily patient questionnaires (Appendix 1). The duration of evaluation was 12 weeks. This questionnaire included four questions about diarrhea, oral mucositis, paronychia and skin conditions based on Common Terminology Criteria for Adverse Events (CTCAE) grading. Furthermore, the number of doses of loperamide per day was also described in this questionnaire. The antitumor efficacy of afatinib was evaluated as a secondary endpoint, including the objective response rate (ORR), PFS, and overall survival (OS). The tumor response was evaluated using Response Evaluation Criteria in Solid Tumors (RECIST), version 1.1 (9). PFS was defined as the time from study enrollment to the date of initial disease progression or death. OS was defined as the time from study enrollment to death from any cause. All of the statistical analysis were performed using JMP 9 statistical software for Macintosh (SAS Institute Inc., Cary, North Carolina, USA).

\section{Prophylactic treatments}

Every eligible patient was treated with prophylactic treatments from the initiation of afatinib monotherapy until disease progression or intolerable toxicity. Treatment for diarrhea: patients were treated with loperamide $2 \mathrm{mg}$ orally twice per day. Treatment for rash and paronychia: patients were treated with minocycline $100 \mathrm{mg}$ orally once per day. Additionally, patients were treated with mediumgrade topical steroids on the body surface, including the fingertips. Treatment for oral mucositis: patients gargled a 
Table 1 Patient characteristics

\begin{tabular}{|c|c|c|c|c|}
\hline Parameter & Subtypes & ITT (n=46), n (\%) & PP (n=35), n (\%) & $1^{\text {st }}$ line $(n=28), n(\%)$ \\
\hline Sex & Female & 32 (69.6) & $25(71.4)$ & $18(64.3)$ \\
\hline Age, years & Median [range] & 65 [36-83] & 65 [36-79] & $65[36-77]$ \\
\hline \multirow[t]{2}{*}{ ECOG-PS } & 0 & $19(41.3)$ & $14(20.0)$ & $12(42.9)$ \\
\hline & 2 & 5 (10.9) & $3(8.6)$ & $4(14.2)$ \\
\hline \multirow[t]{3}{*}{ Histology } & Adenocarcinoma & $43(93.5)$ & $32(91.4)$ & $27(96.4)$ \\
\hline & Squamous & $2(4.3)$ & $2(5.7)$ & $1(3.6)$ \\
\hline & Not other specified & $1(2.2)$ & $1(2.9)$ & 0 \\
\hline Clinical stage & Recurrence & $11(23.9)$ & $11(31.4)$ & $8(31.4)$ \\
\hline \multirow[t]{3}{*}{ EGFR mutation } & Exon 19 deletion & $24(52.2)$ & $15(42.9)$ & $19(42.9)$ \\
\hline & Exon 21 L858R & $11(23.9)$ & $11(31.4)$ & $2(31.4)$ \\
\hline & Others & $11(23.9)$ & $9(25.7)$ & $7(25.7)$ \\
\hline \multirow[t]{3}{*}{ Treatment lines } & $1^{\text {st }}$ line & $28(60.9)$ & $21(60.0)$ & $28(100.0)$ \\
\hline & $2^{\text {nd }}$ line & $7(15.2)$ & $6(17.1)$ & 0 \\
\hline & $\geq 3^{\text {rd }}$ line & $11(23.9)$ & 8 (22.9) & 0 \\
\hline
\end{tabular}

ITT, intention-to treat; PP, per-protocol; ECOG, Eastern Cooperative Oncology Group; PS, performance status.

solution of sodium azulene four times a day.

\section{Results}

\section{Patient characteristics}

Between January 2015 and September 2016, 46 patients were enrolled in this study from 10 institutions. The details of patient enrollment and the analysis population are shown in Figure 1. Eleven enrolled patients were excluded from the primary endpoint analysis for the following reasons: 7 patients required discontinuation or dose reduction of afatinib with AEs other than diarrhea within 4 weeks, 2 patients experienced protocol violation, 1 patient experienced disease progression within 4 weeks, and 1 patient withdrew consent. Primary endpoint analysis was performed in 35 patients (the PP population).

The patient characteristics of each analysis set are presented in Table 1. The distributions of each patient background were similar. In the ITT population, 14 of 46 patients (30.4\%) were male, with a median age of 65 years. The most common tumor histology was adenocarcinoma (93.5\%). EGFR mutations were the mainly common mutations [exon 19 deletion (52.2\%) and exon 21 L858R point mutation (23.9\%)], and 11 patients had uncommon mutations. Twenty-eight patients $(60.9 \%)$ were treated with afatinib as $1^{\text {st }}$-line therapy ( $1^{\text {st }}$-line population).

\section{AEs during the evaluation period in the ITT population}

The AEs that occurred during the evaluation period (84 days after afatinib administration) are summarized in Table 2. The most common AEs were diarrhea, oral mucositis, paronychia and rash/acne. The frequencies of all grades of diarrhea, oral mucositis, paronychia and rash were $83 \%, 57 \%, 52 \%$, and $50 \%$, respectively. The frequencies of grade $\geq 3$ diarrhea, oral mucositis, paronychia and rash were $17 \%, 2 \%, 4 \%$, and $4 \%$, respectively. Treatment discontinuation related to afatinib occurred in 6 patients due to grade 4 transaminase elevation ( 2 patients), grade 3 
Table 2 Adverse events and reason of dose reduction or discontinuation* $(n=46)$

\begin{tabular}{|c|c|c|c|c|}
\hline Adverse event & All grade, $n$ [\%] & Grade 2, n [\%] & $\geq$ Grade 3, n [\%] & Reason of dose, reduction, $\mathrm{n}[\%]$ \\
\hline Oral mucositis & $26[57]$ & 10 [22] & $1[2]$ & $2[4]$ \\
\hline Paronychia & $24[52]$ & $12[26]$ & $2[4]$ & $10[22]$ \\
\hline Rash/acne & $23[50]$ & $6[16]$ & $2[4]$ & $6[13]$ \\
\hline AST elevation & $11[24]$ & $1[2]$ & $2[4]$ & $0[0]$ \\
\hline Anemia & 10 [22] & $4[9]$ & $0[0]$ & $0[0]$ \\
\hline Serum creatinine elevation & $10[22]$ & $1[2]$ & $0[0]$ & $0[0]$ \\
\hline Nausea & $8[17]$ & $4[9]$ & $3[7]$ & $2[4]$ \\
\hline ALT elevation & $8[17]$ & $1[2]$ & $2[4]$ & $0[0]$ \\
\hline Constipation & $8[17]$ & $1[2]$ & $0[0]$ & $0[0]$ \\
\hline Leukopenia & $6[13]$ & $3[7]$ & $1[2]$ & $0[0]$ \\
\hline Neutropenia & $5[11]$ & $0[0]$ & $1[2]$ & $1[2]$ \\
\hline
\end{tabular}

*, adverse events were shown for the ITT population reported in 10\% of patients or more. AST; aspartate transaminase, ALP; alkaline phosphatase, ALT; alanine transaminase.

ileitis, grade 2 paronychia, grade 2 decreased appetite and grade 2 diarrhea. Three patients experienced grade 2 AEs and discontinued afatinib because they could not tolerate the AEs. AEs leading to dose reduction were observed in 27 patients. The main reasons for dose reduction were as follows: diarrhea (12 patients), paronychia (10 patients), decreased appetite (10 patients), rash/acne (6 patients), oral mucositis ( 2 patients), nausea (2 patients) and neutropenia (one patient) (Table 2). Treatment-related death was not observed. The time to AEs in the ITT population is shown in Table 3. The median time to onset of diarrhea, rash/ acne or paronychia due to afatinib was 6,12 or 26 days, respectively.

\section{Primary endpoint analysis in the PP population}

Primary endpoint analysis was performed in 35 patients classified as the PP population. The 4-week successful prophylaxis rate of severe or intolerable diarrhea was 82.9\% (90\% confidence interval: 70.1-91.9\%). While the frequency of severe diarrhea tended to decrease, prophylactic oral loperamide therapy did not demonstrate statistically significant effectiveness. The 4-week successful prophylaxis rate of severe or intolerable oral mucositis, paronychia or rash/acne was $95.6 \%, 100 \%$, or $97.8 \%$, respectively.

\section{Antitumor effect of afatinib}

Antitumor efficacy analyses were performed for 28 patients who were treated in the $1^{\text {st }}$-line setting of the ITT population. The EGFR mutation status was as follows: exon 19 deletion $(\mathrm{n}=19)$, exon $21 \mathrm{~L} 858 \mathrm{R}$ point mutation $(\mathrm{n}=2)$, and uncommon mutation $(n=7)$. The median follow-up duration was 14.8 months. Two patients achieved a complete response, and 21 patients achieved a partial response, for an ORR of $82 \%$. Two patients showed stable disease, for a DCR of $89 \%$. The median PFS was 15.8 months (95\% CI: 8.0-not reach), and the median OS was 26.4 months ( $95 \%$ CI: 26.4-32.1) (Figure 2A,B).

\section{Discussion}

This is the first prospective study to elucidate the efficacy of multimodal prophylactic treatments for afatinib-induced toxicity, including diarrhea, rash, oral mucositis and 
Table 3 Time to adverse events in the ITT population

\begin{tabular}{lcccc}
\hline Variables & $\mathrm{n}$ & $\leq 28$ days, $\mathrm{n}(\%)$ & $>28$ days, $\mathrm{n}(\%)$ & Median time-to-onset of AE \\
\hline Diarrhea & 38 & $38[100]$ & $0[0]$ & $6[2-20]$ \\
Oral mucositis & 26 & $22[85]$ & $4[15]$ & $10[1-374]$ \\
Paronychia & 24 & $13[54]$ & $11[46]$ & $26[2-118]$ \\
Rash/acne & 23 & $20[87]$ & $3[13]$ & $12[4-58]$ \\
Decreased appetite & 20 & $18[90]$ & $2[10]$ & $9[2-57]$ \\
AST elevation & 11 & $11[79]$ & $3[21]$ & $17[2-489]$ \\
Anemia & 10 & $9[75]$ & $3[25]$ & $14[2-76]$ \\
Serum creatinine elevation & 10 & $10[91]$ & $1[9]$ & $11[2-64]$ \\
Nausea & 8 & $7[88]$ & $1[12]$ & $5.5[2-30]$ \\
ALP elevation & 8 & $7[78]$ & $2[22]$ & $12[2-489]$ \\
Dry skin & 8 & $5[63]$ & $3[37]$ & $11[4-83]$ \\
ALT elevation & $7[70]$ & $3[30]$ & $14[2-489]$ \\
Constipation & 8 & $10[100]$ & $0[0]$ & $2[1-6]$ \\
Leukopenia & 8 & $1[14]$ & $17[7-76]$ \\
Neutropenia & 6 & $2[46]$ & $28[14-57]$ \\
\hline
\end{tabular}

$\mathrm{ITT}$, intention-to treat; AE, adverse event; AST, aspartate transaminase; ALP, alkaline phosphatase; ALT, alanine transaminase.
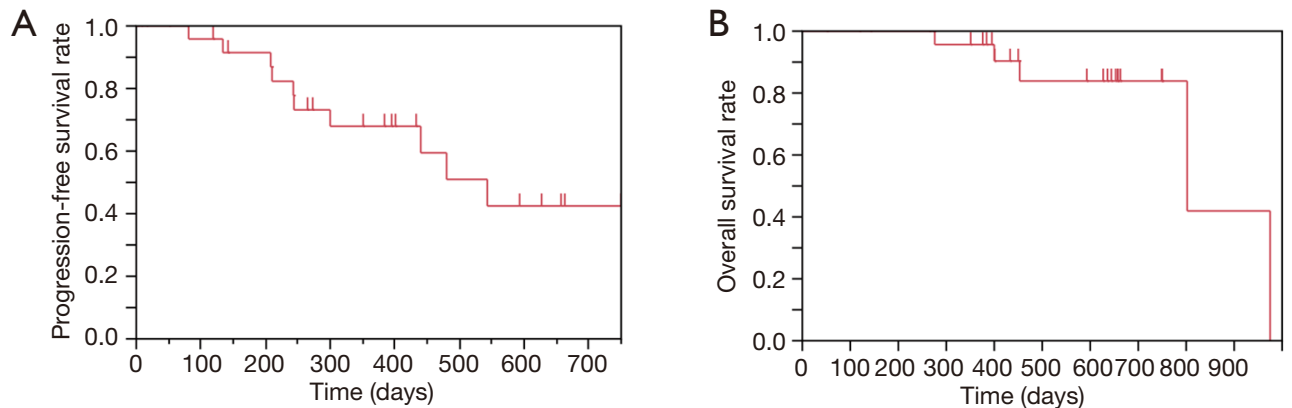

Figure 2 Survival analyses for $1^{\text {st }}$-line afatinib (n=28). (A) Kaplan-Meier curve of progression-free survival (PFS). The median PFS duration was 15.8 months. (B) Kaplan-Meier curve of overall survival (OS). The median OS duration was 26.4 months.

paronychia. This study could not demonstrate efficacy of prophylactic loperamide use for preventing severe diarrhea induced by afatinib. However, multimodal treatment including minocycline with medium-grade topical steroid and gargling with a solution of sodium azulene might be effective for preventing other problematic AEs. These kinds of interventions can improve the tolerability of a $40 \mathrm{mg}$ dose of afatinib and impact the antitumor effect.

Afatinib is a second-generation EGFR-TKI and an irreversible inhibitor of ErbB family members, including
EGFR. This agent is potentially effective compared with first-generation EGFR-TKIs. However, many patients treated with afatinib experienced severe or intolerable AEs, such as diarrhea, oral mucositis, paronychia, or rash/acne. In two pivotal phase III trials of afatinib (LUX-Lung 3 and LUX-Lung 6), the dose reduction rates due to AEs were $52 \%$ and $28 \%$, respectively $(1,2)$. In the Japanese subgroup analysis of the LUX-Lung 3 trial, the incidence of all grade or grade 3 and higher AEs was higher than that in the global population (8). The improvement of afatinib-induced 
$\mathrm{AE}$ management remains an important issue.

Afatinib-induced diarrhea is the most problematic AE and often affects quality of life. In the Japanese subgroup analysis of the LUX-Lung 3 trial, the incidence of all grade or grade 3 and higher diarrhea was $100 \%$ or $22.2 \%$, respectively (8). The time to onset of diarrhea was 4 days, with a range of 2-84 days (10). It remains an important clinical question whether the use of prophylactic antidiarrheal drugs prevents severe diarrhea induced by afatinib. The current study investigated the prophylactic effectiveness of the use of loperamide $2 \mathrm{mg}$ orally twice per day for afatinib-induced diarrhea. The incidence of all grade or grade 3 and higher diarrhea was $83 \%$ or $17 \%$, respectively. The median time to onset of diarrhea was 6 days, with a range of 2-20 days. These data suggested that aggressive use of loperamide might be effective for preventing severe diarrhea and delaying the time to onset of diarrhea compared with the data of Japanese subgroup analysis of LUX-Lung 3. However, the effectiveness of aggressive use of loperamide was marginal, and constipation was frequently observed in this study (the all-grade frequency was $22 \%$, and the grade 2 frequency was $4 \%$ ). The prophylactic administration of loperamide should not be recommended to all patients who are treated with afatinib.

Few prospective studies have investigated an antidiarrheal drug for afatinib-induced diarrhea. The prospective phase II study to evaluate the preventive effect of kampo medicine (hangeshashinto, TJ-14) for afatinib-induced diarrhea reported that the incidence of $\geq$ grade 3 diarrhea within 4 weeks of treatment was $3.4 \%$ (11). However, kampo medicine has not been recognized as the global standard treatment because of insurance reimbursement or unexpected side effects, such as interstitial lung disease. A pharmacokinetics study of afatinib demonstrated that afatinib-induced severe diarrhea was associated with the maximum plasma concentration values and the area under the plasma concentration-time curve (12). However, the prediction of severe diarrhea will be very difficult because afatinib plasma levels showed interpatient variability in the pharmacokinetic study of the LUX-Lung 3 trial (1). The post hoc analysis of LUX-Lung 3 and 6 suggested that symptom-based dose reduction was efficacious for reaching the adequate afatinib dosage (4). The randomized phase IIb LUX-Lung 7 study reproducibly showed the similar efficacy of afatinib in patients who experienced dose reduction due to treatment related adverse event compared with patients who could treat with $40 \mathrm{mg}$ dose once a day (13).
Considering these reports and the result of our study that prophylactic loperamide administration was not effective in preventing afatinib-induced severe diarrhea, not hesitating to perform a dose reduction according to the patient's symptoms is the appropriate practical approach to manage afatinib-induced diarrhea.

Skin toxicities, including rash/acne and paronychia, were common AEs in EGFR-TKI therapy. In the Japanese subgroup analysis of the LUX-Lung 3 trial, the incidence of all grade or grade 3 and higher rash/acne was $100 \%$ or $20.4 \%$, respectively (8). The incidence of all grade or grade 3 and higher nail effects (paronychia) was $92.6 \%$ or $25.9 \%$, respectively (8). The time to onset of rash/acne or paronychia was 7 days (range, 1-171 days) or 37 days (range, 8-278 days), respectively (10). The preventive efficacy of tetracyclines and topical corticosteroids for EGFR-TKI-induced rashes has been investigated. A prospective randomized phase III trial showed that 4 weeks of prophylactic minocycline administration could reduce the severity of erlotinib-induced skin rash (7). It has been suggested that prophylactic tetracycline will be a promising strategy to prevent severe skin rash induced by EGFR-TKIs. In the current study, the incidence of severe rash and acne or paronychia was only $4 \%$. The current study demonstrated that a prophylactic approach using minocycline administration with topical corticosteroids reduced the incidence of afatinib-induced dermatologic AEs but did not delay the time to onset of afatinib-induced dermatologic AEs. Notably, paronychia is the most common toxicity that compromises quality of life due to pain in patients treated with afatinib. However, the management of these AEs has not been fully investigated. This study is the first to report the possibility of preventive efficacy of minocycline with topical corticosteroids for afatinib-induced paronychia. On the other hand, prophylactic minocycline might induce additional side effects. Two cases of severe transaminase elevation were reported in the current study. Severe liver injury is a rare AE in afatinib therapy because afatinib is mainly metabolized by non-enzymecatalyzed Michael adduct formation on proteins (14). Long-term minocycline use may be a risk factor for liver injury, including autoimmune hepatitis (15). During prophylactic use of minocycline administration for afatinibinduced rash/acne or paronychia, attention should be paid to liver injury, and it is necessary to refrain from long-term administration.

Patients treated with afatinib often experience oral mucositis. Patients who experience this AE suffer from 
dysgeusia and oral pain, but an effective prophylactic strategy has not been established. Basic oral care is essential to prevent oral mucositis, but further intervention will be needed for chemotherapy- or EGFR-TKI-induced oral mucositis (16). Gargling a solution of sodium azulene is often carried out empirically for the prevention of oral mucositis, but there are few prospective data. The incidence of severe oral mucositis in this study was only $2 \%$. This is the first prospective study to evaluate the preventive efficacy of gargling a solution of sodium azulene for afatinibinduced oral mucositis.

The limitation of this study is that these results were depended on a small population-based nonrandomized trial, and the primary endpoint was not met. Thus, we recommend a dose reduction to manage the afatinibinduced severe diarrhea, not a prophylactic and aggressive use of loperamide. Furthermore, the sample size of this study was relatively small regarding evaluating the secondary endpoints. However, the result of this study suggests that multimodal intervention should consists of minocycline, topical steroids and gargling with sodium azulene as useful in preventing severe toxicities due to afatinib and in maintaining the compliance of afatinib. Notably, the median PFS was 15.8 months, and the median OS was 26.4 months in the efficacy analysis. These results were obviously favorable compared with previously reported data. To confirm the efficacy of this multimodal prophylactic treatment for afatinib therapy, a randomized trial is warranted.

\section{Conclusions}

Prophylactic loperamide was not effective in preventing severe or intolerable diarrhea within 4 weeks during afatinib treatment. The appropriate management for severe diarrhea induced by afatinib is tolerability-guided dose adjustment. Multimodal prevention using minocycline, topical steroids and gargling with sodium azulene has the potential to maintain compliance with afatinib.

\section{Acknowledgments}

We thank the patients and their families for their support and participation in this study.

Funding: This work was supported by Niigata University Hospital Clinical Research Aid (CRA2015-03).

\section{Footnote}

Reporting Checklist: The authors have completed the TREND reporting checklist. Available at http://dx.doi. org/10.21037/tlcr-20-649

Data Sharing Statement: Available at http://dx.doi. org/10.21037/tlcr-20-649

Conflicts of Interest: All authors have completed the ICMJE uniform disclosure form (available at http://dx.doi. org/10.21037/tlcr-20-649). SM has received lecture fees from Chugai Pharmaceutical, ONO Pharmaceutical, AstraZeneca, Eli Lilly, MSD, and Nippon Boehringer Ingelheim. SW has received lecture fees from Chugai Pharmaceutical, AstraZeneca. HT has received lecture fees from Chugai Pharmaceutical, AstraZeneca, Nippon Boehringer Ingelheim, and Bristol-Myers Squibb. Toshiaki Kikuchi has received lecture fees from Chugai Pharmaceutical, Nippon Boehringer Ingelheim, AstraZeneca, Pfizer Japan, DAIICHI SANKYO. TK has received research funds from AstraZeneca K.K. Toshiaki Kikuchi has received research grants from Chugai Pharmaceutical, ONO Pharmaceutical, Eli Lilly Japan, Nippon Boehringer Ingelheim. The other authors have no conflicts of interest to declare.

Etbical Statement: The authors are accountable for all aspects of the work in ensuring that questions related to the accuracy or integrity of any part of the work are appropriately investigated and resolved. This study was performed in accordance with the Helsinki Declaration (as revised in 2013). The Institutional Review Board of each participating institution approved the protocol. This study was registered in January 2015 on the clinical trial site of the University Hospital Medical Information Network Clinical Trials Registry (registration number: UMIN000016167).

Open Access Statement: This is an Open Access article distributed in accordance with the Creative Commons Attribution-NonCommercial-NoDerivs 4.0 International License (CC BY-NC-ND 4.0), which permits the noncommercial replication and distribution of the article with the strict proviso that no changes or edits are made and the original work is properly cited (including links to both the formal publication through the relevant DOI and the license). 
See: https://creativecommons.org/licenses/by-nc-nd/4.0/.

\section{References}

1. Sequist LV, Yang JC, Yamamoto N, et al. Phase III study of afatinib or cisplatin plus pemetrexed in patients with metastatic lung adenocarcinoma with EGFR mutations. J Clin Oncol. 2013;31:3327-34.

2. Wu YL, Zhou C, Hu CP, et al. Afatinib versus cisplatin plus gemcitabine for first-line treatment of Asian patients with advanced non-small-cell lung cancer harbouring EGFR mutations (LUX-Lung 6): an open-label, randomised phase 3 trial. Lancet Oncol 2014;15:213-22.

3. Yang JC, Wu YL, Schuler M, et al. Afatinib versus cisplatin-based chemotherapy for EGFR mutation-positive lung adenocarcinoma (LUX-Lung 3 and LUX-Lung 6): analysis of overall survival data from two randomised, phase 3 trials. Lancet Oncol 2015;16:141-51.

4. Park K, Tan EH, O'Byrne K, et al. Afatinib versus gefitinib as first-line treatment of patients with EGFR mutationpositive non-small-cell lung cancer (LUX-Lung 7): a phase 2B, open-label, randomised controlled trial. Lancet Oncol 2016;17:577-89.

5. Ding PN, Lord SJ, Gebski V, et al. Risk of TreatmentRelated Toxicities from EGFR Tyrosine Kinase Inhibitors: A Meta-analysis of Clinical Trials of Gefitinib, Erlotinib, and Afatinib in Advanced EGFR-Mutated Non-Small Cell Lung Cancer. J Thorac Oncol 2017;12:633-43.

6. Felip E, Hirsh V, Popat S, et al. Symptom and Quality of Life Improvement in LUX-Lung 8, an Open-Label Phase III Study of Second-Line Afatinib Versus Erlotinib in Patients With Advanced Squamous Cell Carcinoma of the Lung After First-Line Platinum-Based Chemotherapy. Clin Lung Cancer 2018;19:74-83.e11.

7. Melosky B, Anderson H, Burkes RL, et al. Pan Canadian Rash Trial: A Randomized Phase III Trial Evaluating the Impact of a Prophylactic Skin Treatment Regimen on Epidermal Growth Factor Receptor-Tyrosine Kinase Inhibitor-Induced Skin Toxicities in Patients With Metastatic Lung Cancer. J Clin Oncol 2016;34:810-5.

8. Kato T, Yoshioka H, Okamoto I, et al. Afatinib versus cisplatin plus pemetrexed in Japanese patients with advanced non-small cell lung cancer harboring activating EGFR mutations: Subgroup analysis of LUX-Lung 3.
Cancer Sci 2015;106:1202-11.

9. Eisenhauer EA, Therasse P, Bogaerts J, et al. New response evaluation criteria in solid tumours: revised RECIST guideline (version 1.1). Eur J Cancer 2009;45:228-47.

10. Afatinib Maleate Proper Use Guide. Available online: http://www.bij-kusuri.jp/products/attach/pdf/gio_t_guide. pdf. 2016.

11. Ichiki M, Wataya H, Yamada K, et al. Preventive effect of kampo medicine (hangeshashin-to, TJ-14) plus minocycline against afatinib-induced diarrhea and skin rash in patients with non-small cell lung cancer. Onco Targets Ther 2017;10:5107-13.

12. Wind S, Schmid M, Erhardt J, et al. Pharmacokinetics of afatinib, a selective irreversible ErbB family blocker, in patients with advanced solid tumours. Clin Pharmacokinet 2013;52:1101-9.

13. Schuler M, Tan EH, O'Byrne K, et al. First-line afatinib vs gefitinib for patients with EGFR mutationpositive NSCLC (LUX-Lung 7): impact of afatinib dose adjustment and analysis of mode of initial progression for patients who continued treatment beyond progression. J Cancer Res Clin Oncol 2019;145:1569-79.

14. Freiwald M, Schmid U, Fleury A, et al. Population pharmacokinetics of afatinib, an irreversible ErbB family blocker, in patients with various solid tumors. Cancer Chemother Pharmacol 2014;73:759-70.

15. Lawrenson RA, Seaman HE, Sundström A, et al. Liver damage associated with minocycline use in acne: a systematic review of the published literature and pharmacovigilance data. Drug Saf 2000;23:333-49.

16. Lalla RV, Bowen J, Barasch A, et al. MASCC/ISOO clinical practice guidelines for the management of mucositis secondary to cancer therapy. Cancer 2014;120:1453-61.

Cite this article as: Okajima $\mathrm{M}$, Miura $\mathrm{S}$, Watanabe $\mathrm{S}$, Tanaka H, Ito K, Ishida T, Makino M, Iwashima A, Matsumoto N, Sato K, Ichikawa K, Abe T, Yoshizawa H, Kikuchi T. A prospective phase II study of multimodal prophylactic treatment for afatinib-induced adverse events in advanced non-small cell lung cancer (Niigata Lung Cancer Treatment Group 1401). Transl Lung Cancer Res 2021;10(1):252260. doi: $10.21037 /$ tlcr-20-649 


\section{Symptom Diary (NLCTG1401)}

Patients treated with Giotrif often suffer from diarrhea, stomatitis, skin rash and paronychia. Please describe your daily symptoms.

Please complete this questionairre everyday. (No need to fill in the first day of treatment.)

If you have any questions about this questionnaire, please contact your doctor.

When you have completed this form, please put it in a sealed envelope and give it to your doctor.

identification number : 


\section{Day $\mathrm{O}$ of Giotrif $\quad$ (ID:}

Date:

(1) How many stools have you had in the 24 hours since yesterday morning?

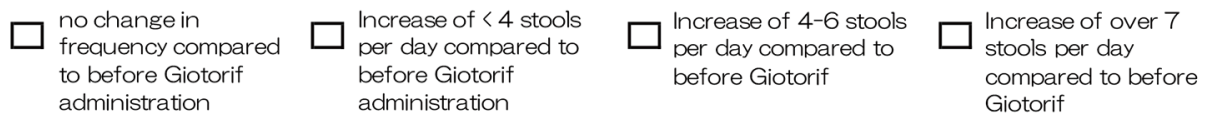

(2) How many Loperamid capsules did you take in the 24 hours since yesterday morning?

( ) capsules (Fill out the form, including the amount you take on a regular basis (2 capsules in the morning).)

(3) Please tell me about your oral conditions

$\square$ none $\quad \square \begin{aligned} & \begin{array}{l}\text { Slightly pain; } \\ \text { intervention not } \\ \text { required }\end{array} \\ & \begin{array}{l}\text { Modarate pain; } \\ \text { interfering with oral } \\ \text { intake }\end{array}\end{aligned} \quad \begin{aligned} & \text { Severe pain; } \\ & \text { interfering with oral } \\ & \text { intake }\end{aligned}$

(4) Please tell me about your nail conditions.

intake
No change from
Nail fold edema or
pre-treatment.
erythema
Nail fold edema or
erythema with pain;
Limiting instrumental
ADL
Nail fold edema or
erythema with severe
pain; Limiting selfcare
ADL

(5)Please tell me about your skin conditions.

$\square \begin{aligned} & \text { No change from } \\ & \text { pre-treatment. }\end{aligned}$

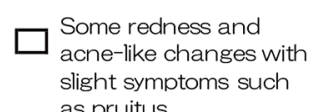

Symptomatic skin

changes such as

papules and/or

pustules:

Limiting instrumental

$\square$ Symptomatic skin

changes such as papules and/or pustules;

Limiting selfcare ADL

ADL

\section{Day $\bigcirc \bigcirc$ of Giotrif (ID: )}

Date ;

(1) How many stools have you had in the 24 hours since yesterday morning?

\begin{tabular}{|c|c|c|c|}
\hline $\begin{array}{l}\text { no change in } \\
\text { frequency compared } \\
\text { to before Giotorif } \\
\text { administration }\end{array}$ & $\begin{array}{l}\text { Increase of }<4 \text { stools } \\
\text { per day compared to } \\
\text { before Giotorif } \\
\text { administration }\end{array}$ & $\begin{array}{l}\text { Increase of } 4-6 \text { stools } \\
\text { per day compared to } \\
\text { before Giotorif } \\
\text { administration }\end{array}$ & $\begin{array}{l}\text { Increase of over } 7 \\
\text { stools per day } \\
\text { compared to before } \\
\text { Giotorif }\end{array}$ \\
\hline
\end{tabular}

(2) How many Loperamid capsules did you take in the 24 hours since yesterday morning?

( ) capsules (Fill out the form, including the amount you take on a regular basis (2 capsules in the morning).)

(3Please tell me about your mouth conditions

none

$\square$
Slightly pain;

intervention not

required
Modarate pain;

that does not

interfering with oral

intake
Severe pain;

interfering with oral

intake

(4) Please tell me about your nail conditions.

$\square$ No change from

pre-treatment.

Nail fold edema or
erythema
Nail fold edema or erythema with pain; Limiting instrumental ADL
Nail fold edema or erythema with severe pain; Limiting selfcare

(5) Please tell me about your skin conditions.

No change from pre-treatment.
Some redness and acne-like changes with slight symptoms such as pruitus.
Symptomatic skin changes such as papules and/or pustules:

Limiting instrumental

ADL
Symptomatic skin

changes such as papules and/or pustules: 